Article

\title{
Biodiesel by Transesterification of Rapeseed Oil Using Ultrasound: A Kinetic Study of Base-Catalysed Reactions
}

\author{
José María Encinar $^{1, *(\mathbb{D})}$, Ana Pardal ${ }^{2}$ (D), Nuria Sánchez ${ }^{1}$ and Sergio Nogales 1 (D) \\ 1 Department of Chemical Engineering and Physical Chemistry, Extremadura University, \\ Avenida de Elvas s/n., 06071 Badajoz, Spain; nuriass@unex.es (N.S.); senogalesd@unex.es (S.N.) \\ 2 Department of Applied Sciences and Technology, IP Beja, Rua Pedro Soares s/n., 7800 Beja, Portugal; \\ anap@ipbeja.pt \\ * Correspondence: jencinar@unex.es; Tel.: +34-924-289-672
}

Received: 27 July 2018; Accepted: 23 August 2018; Published: 25 August 2018

\begin{abstract}
The objective of this work was to study the acceleration that ultrasound causes in the rate of biodiesel transesterification reactions. The effect of different operating variables, such as ultrasound power, catalyst $(\mathrm{KOH})$ concentration and methanol:oil molar ratio, was studied. The evolution of the process was followed by gas chromatography, determining the concentration of methyl esters at different reaction times. The biodiesel was characterized by its density, viscosity, saponification and iodine values, acidity index, water content, flash and combustion points, cetane index and cold filter plugging point (CFPP), according to EN 14214 standard. High methyl ester yield and fast reaction rates were obtained in short reaction times. Ultrasound power and catalyst concentration had a positive effect on the yield and the reaction rate. The methanol:oil molar ratio also increased the yield of the reaction, but negatively influenced the process rate. The reaction followed a pseudo-first order kinetic model and the rate constants at several temperatures were determined. The activation energy was also determined using the Arrhenius equation. The main conclusion of this work is that the use of ultrasound irradiation did not require any additional heating, which could represent an energy savings for biodiesel manufacture.
\end{abstract}

Keywords: fatty acid methyl ester; catalyst; viscosity; iodine value; acidity index

\section{Introduction}

The future development of world economy makes finding renewable sources of energy that can replace fossil fuels necessary. For years, biodiesel has been a real alternative to fossil fuels used in internal combustion engines [1]. As it is known, biodiesel is a fuel consisting of monoalkyl esters of long-chain fatty acids (FAME) derived from renewable lipid feedstocks, and it is generally produced via transesterification [2,3]. A lot of different raw materials have been used to obtain biodiesel. Edible vegetable oils such as canola and soybean oil in the USA, palm oil in Malaysia or rapeseed oil in Europe have been used for biodiesel production and found to be good substitutes for diesel. Non-edible vegetable oils, such as Pongamia pinnata (karanja or honge), Jatropha curcas (jatropha or ratanjyote), Citrus reticulata (mandarin) and Madhuca iondica (mahua) have also been found to be suitable for biodiesel production [4-6]. Concerning the different types of vegetable oils and their composition, fatty acids with high unsaturation levels usually imply lower fluidity at low temperatures, leading to solidification. Thus, oils with a high ratio of monounsaturated fatty acid (and a low ratio of polyunsaturated fatty acids) usually show good performance at low temperatures. As rapeseed oil has such a composition, that is the reason why it is frequently used for biodiesel production in Europe. 
Rapeseed oil usually contains around $98 \%$ of triglycerides. The main components of this oil are oleic acid (monounsaturated), that is present in more than $60 \%$, and linoleic acid (doubly unsaturated) that exceeds $20 \%$ [4]. For these reasons, rapeseed oil was chosen to carry out this work.

Although there are other possibilities, transesterification has been reported as the most common way to produce biodiesel from vegetable oil. Alcohols such as methanol or ethanol are the more frequently used alcohols. A catalyst is necessary to increase the reaction rate and the conversion yield. The catalysts used can be homogeneous and heterogeneous and they can be acid or basic in nature. Generally, basic homogenous catalysis is the preferred option to provide high reaction rates. Additionally, basic catalysts are readily available and very cheap. In the literature there is comprehensive information about the catalytic processes involved [3,7-9].

The mechanism of the transesterification of vegetable oils by means of basic catalysis is well known and published $[10,11]$. The mechanism comprises four stages. In the first step the base (catalyst) reacts with the alcohol, giving an alkoxide and the protonated catalyst. The second step consists in the nucleophilic attack of the alkoxide at the carbonyl group of the triglyceride, generating the alkyl ester and the corresponding anion of the diglyceride (third step). For the final step, this diglyceride deprotonates the catalyst, making it active and able to react with another alcohol, starting a new transtererification cycle. Diglycerides and monoglycerides are equally converted (to a mixture of alkyl esters and glycerol) by this mechanism.

The transesterification reaction is initially heterogeneous because methanol is only partially miscible with triglycerides at room temperature. For this reason, the reaction initially is slow and only takes place at the alcohol-oil interphase, and the process is dependent of mass transfer. When the stirring rate is high, emulsions are usually generated. These emulsions are caused, mainly, by the intermediate monoglycerides and diglycerides, which have both polar (hydroxyl groups) and non-polar (hydrocarbon chains) parts. Therefore, when a critical concentration of these intermediates is exceeded, emulsion takes place. Due to the low miscibility between methanol, vegetable oils and methyl esters, this emulsion is not stable and breaks. The use of ultrasonic irradiation could avoid this problem. Ultrasound produces special chemical and physical effects due to the collapse of cavitation bubbles. Low frequency sound waves can produce emulsions between immiscible liquids, being useful for the transesterification of triglycerides with alcohol [12-14]. Ultrasound has several effects on transesterification: acoustic streaming mixing or changes in sound pressure, leading to fast movement of fluids and cavitation bubbles that apply negative pressure gradients on liquids. The most important effect is the formation and collapse of cavitation bubbles, providing high temperature and pressure, with a significant influence on reaction rates, mass transfer and catalytic surface areas. As a consequence, the use of sonochemical reactors can favor the chemical reaction and propagation by way of enhanced mass transfer and interphase mixing between the phases and also can lower the requirements of the operating conditions (in terms of temperature and pressure) [15].

The main advantages of ultrasonic irradiation process are: shorter reaction times, lower alcohol/oil molar ratios, less energy consumption (50\%), lower concentration of catalyst, higher reaction rate and conversion, improved yield, simpler separation and purification processes, and higher quality glycerol production $[7,9,11,14]$.

In contrast, this process has some disadvantages such as: the reaction temperature was slightly higher for long reactions and the ultrasonic power must be under control due to the possibility of soap formation in fast reactions [16]. Nevertheless, as it is indicated, energetically the process is highly favorable, because of the formation of micro jets and neither localized temperature increases no agitation or heating are required to produce biodiesel with ultrasound application [17], therefore, according to the literature, the beneficial effect of ultrasound is due to the generation of a fine emulsion between methanol and fatty acids, increasing the surface area for the necessary chemical reactions. No adverse effects, such as the generation of free radicals and the subsequent chain reactions caused by them, were observed [18]. Thus, the use of ultrasound could imply an improvement in biodiesel production, increasing the yield of the product. 
Therefore, it could be said that ultrasound radiation provides energy to form a mixture and overcome the energy activation barrier that is necessary for the process. On the other hand, high temperatures and pressures can be achieved locally, which could imply an intense mixture between the reagents. All these circumstances favor the reaction progress.

Considering the abovementioned facts, the objective of this work was to study the $\mathrm{KOH}$-catalysed transesterification reaction of rapeseed oil using an ultrasonic mixing technique, determining the suitable reaction conditions to carry out the process in a short time and with low energy consumption. Also, a kinetic study of the reaction in the presence of ultrasonic radiation was carried out, determining the parameters necessary for the reactor design. In addition, the obtained results were compared to the previously obtained ones in the transesterification of rapeseed oil in the absence of radiation $[19,20]$.

\section{Materials and Methods}

The raw material (rapeseed oil) was provided by the Research Center "La Orden-Valdesequera" (Badajoz, Spain), Section of Non-Edible Crops. The rapeseed oil was characterized by density, viscosity, water content, acid, iodine and saponification values and fatty acid profile (Table 1).

Table 1. Rapeseed oil fatty acid profile and properties.

\begin{tabular}{lc}
\hline Fatty Acid & Percentage (\%) \\
\hline C16:0 Palmitic & 3.5 \\
C18:0 Stearic & 0.9 \\
C18:1 Oleic & 64.4 \\
C18:2 Linoleic & 22.3 \\
C18:3 Linolenic & 8.2 \\
Other minority acids & 0.7 \\
\hline Properties & \\
\hline Density at $15{ }^{\circ} \mathrm{C}\left(\mathrm{kgm}^{-3}\right)$ & 906.2 \\
Viscosity at $40{ }^{\circ} \mathrm{C}(\mathrm{cSt})$ & 36.3 \\
Water content $(\mathrm{wt}, \%)$ & 0.8 \\
Acidity index $\left(\mathrm{mg}_{\mathrm{KOH}} \cdot \mathrm{g}_{\mathrm{oil}}{ }^{-1}\right)$ & 2.7 \\
Iodine value $\left(\mathrm{g}_{\mathrm{I}} \cdot 100 \mathrm{~g}^{-1}\right)$ & 113.5 \\
Saponification value $\left(\mathrm{mg}_{\mathrm{KOH}} \cdot \mathrm{g}_{\mathrm{oil}}{ }^{-1}\right)$ & 194.7 \\
\hline
\end{tabular}

Potassium hydroxide ( $\mathrm{KOH}$, pellets GR for analysis), used as a catalyst, was supplied by Merck (Darmstadt, Germany) and methanol, 99\%, was supplied by Panreac (Castellar del Vallés, Spain). The other chemicals were obtained commercially (Merck) and were of analytical grade. The experimental design is shown in Figure 1. The transesterification was carried out in a $500 \mathrm{~mL}$ spherical reactor, with a temperature sensor, sampling outlet and condensation systems, using a sonicator (Digital Sonifier, model 450, Branson, MO, USA), as shown in Figure 2. This sonicator has a fixed working frequency of $20 \mathrm{kHz}$ and a power of $400 \mathrm{~W}$, with adjustable levels for the latter between 0 and $100 \%$.

Firstly, the reactor was charged with oil. Different amounts of catalyst were dissolved in different amounts of methanol and the resulting solution was added to the reactor. At this point, the sonicator was placed at the different power values and then the reaction started, taking place for 15-20 min. The different reaction conditions are specified in Table 2. 


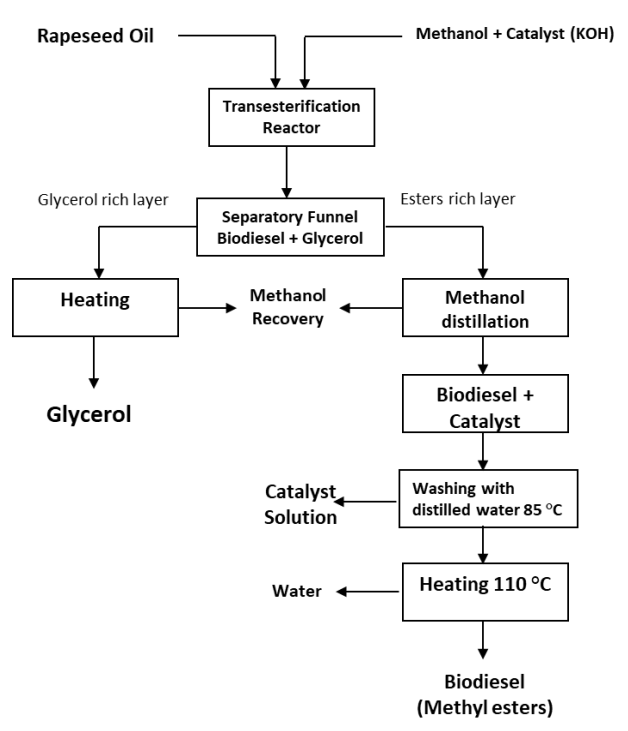

Figure 1. Schematic flow diagram of the biodiesel production from rapeseed oil.

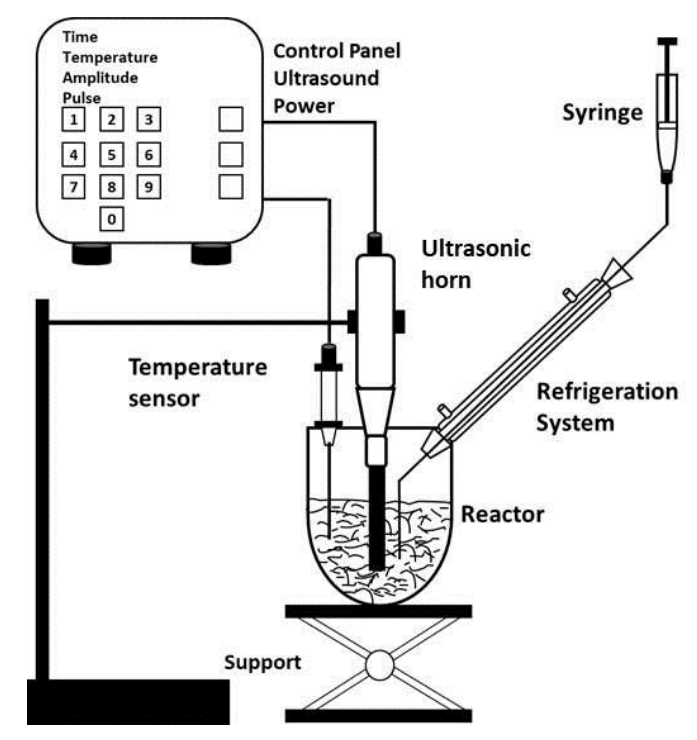

Figure 2. Schematic representation of the experimental setup.

Table 2. Experimental conditions. Properties of biodiesel produced from rapeseed oil and comparison with the EN 14214 standard.

\begin{tabular}{|c|c|c|c|c|c|c|c|}
\hline Run & $\begin{array}{c}\text { MeOH:Oil } \\
\text { Molar Ratio }\end{array}$ & $\begin{array}{c}{ }^{\mathrm{a}} \mathrm{KOH} \\
(\%)\end{array}$ & $\begin{array}{l}\mathrm{b} \text { Ultrasound } \\
\text { Power, \%, (W) }\end{array}$ & $\mathrm{T}_{\max },\left({ }^{\circ} \mathrm{C}\right)$ & ${ }^{c}$ Yield, \% & $\begin{array}{c}\text { Density } 15^{\circ} \mathrm{C}, \\
\mathrm{kg} / \mathrm{m}^{3}\end{array}$ & $\begin{array}{c}\text { Viscosity } \\
40^{\circ} \mathrm{C}, \mathrm{cSt}\end{array}$ \\
\hline 1 & $9: 1$ & 0.7 & $20,(80 \mathrm{~W})$ & $49( \pm 2)$ & $55.6( \pm 1.1)$ & $879.4( \pm 2.1)$ & $8.8( \pm 0.3)$ \\
\hline 2 & $9: 1$ & 0.7 & $40,(160 \mathrm{~W})$ & $60( \pm 2)$ & $86.6( \pm 0.9)$ & $872.0( \pm 2.0)$ & $4.9( \pm 0.2)$ \\
\hline 3 & $9: 1$ & 0.7 & $60,(240 \mathrm{~W})$ & $64( \pm 1)$ & $92.0( \pm 0.7)$ & $867.9( \pm 1.7)$ & $4.8( \pm 0.1)$ \\
\hline 4 & $9: 1$ & 0.7 & $80,(320 \mathrm{~W})$ & $68( \pm 1)$ & $94.9( \pm 0.9)$ & $866.3( \pm 1.2)$ & $4.4( \pm 0.2)$ \\
\hline 5 & $9: 1$ & 0.7 & $100,(400 \mathrm{~W})$ & $70( \pm 2)$ & $96.6( \pm 0.8)$ & $861.9( \pm 1.5)$ & $4.7( \pm 0.2)$ \\
\hline 6 & $9: 1$ & 0.3 & $100,(400 \mathrm{~W})$ & $69( \pm 1)$ & $41.1( \pm 1.6)$ & $888.5( \pm 2.1)$ & $13.9( \pm 0.5)$ \\
\hline 7 & $9: 1$ & 0.5 & $100,(400 \mathrm{~W})$ & $70( \pm 2)$ & $94.0( \pm 1.0)$ & $866.2( \pm 1.3)$ & $4.8( \pm 0.2)$ \\
\hline 8 & $9: 1$ & 1.0 & $100,(400 \mathrm{~W})$ & $70( \pm 2)$ & $93.6( \pm 1.0)$ & $866.5( \pm 0.9)$ & $4.4( \pm 0.1)$ \\
\hline 9 & $3: 1$ & 0.7 & $100,(400 \mathrm{~W})$ & $80( \pm 1)$ & $70.8( \pm 1.3)$ & $875.5( \pm 1.4)$ & $6.9( \pm 0.2)$ \\
\hline 10 & $6: 1$ & 0.7 & $100,(400 \mathrm{~W})$ & $75( \pm 1)$ & $97.4( \pm 1.2)$ & $870.9( \pm 0.8)$ & $4.9( \pm 0.2)$ \\
\hline 11 & $12: 1$ & 0.7 & $100,(400 \mathrm{~W})$ & $70( \pm 1)$ & $98.0( \pm 1.1)$ & $866.5( \pm 1.3)$ & $4.4( \pm 0.1)$ \\
\hline 12 & $9: 1$ & 0.7 & $0,(0 \mathrm{~W})$ & $55( \pm 2)($ ISO $)$ & $61.2( \pm 1.4)$ & $872.0( \pm 1.2)$ & $7.6( \pm 0.2)$ \\
\hline EN-14214 & - & - & - & - & 96.5 & $860-900$ & $3.5-5.0$ \\
\hline
\end{tabular}

a The catalyst percentage is referred to the initial mass of oil; ${ }^{\mathrm{b}}$ The values in parentheses specify the applied power in watts; ${ }^{c}$ The yield is referred to the mass of methyl esters in the total biodiesel mass. 
After an appropriate reaction time, the mixture was placed in a separatory funnel for $24 \mathrm{~h}$ to ensure that the separation of biodiesel and glycerol phases was complete. The glycerol (bottom) phase was removed and left in a container. Methyl esters (biodiesel) were heated, at $85^{\circ} \mathrm{C}$ to remove excess methanol. The remaining catalyst was extracted by successive rinses with distilled water. Finally, traces of water were eliminated by heating at $110^{\circ} \mathrm{C}$.

The evolution of the process (methyl ester content) was followed by gas chromatography on a VARIAN 3900 chromatograph (Varian, Palo Alto, California, USA), provided with an FID, employing a silica capillary column of $30 \mathrm{~m}$ length, $0.32 \mathrm{~mm}$ ID, and $0.25 \mathrm{~mm}$ film thickness. Heptane was used as a solvent, and the carrier gas was helium at a flow rate of $0.7 \mathrm{~mL} / \mathrm{min}$. The injector and the detector temperatures were kept at $270{ }^{\circ} \mathrm{C}$ and $300{ }^{\circ} \mathrm{C}$, respectively. The temperature ramp started at $200{ }^{\circ} \mathrm{C}$, and then increased $20^{\circ} \mathrm{C} / \mathrm{min}$ up to $220^{\circ} \mathrm{C}$. The calibration curve of the peak areas versus the quantity of biodiesel was linear. The samples were taken out from the reaction mixture, neutralized and heated to remove methanol, centrifuged for $5 \mathrm{~min}$ at $6000 \mathrm{rpm}$, and then analyzed by gas chromatography.

Thus, the yield was referred to the mass of FAME in the total biodiesel mass, as follows (Equation (1)):

$$
\text { Yield }(\%)=\frac{\sum m_{i}}{m_{T}} * 100
$$

where $m_{i}$ is the mass of each FAME, and $m_{T}$ is the mass of biodiesel, both in mg. The analytical methods used to determine the characteristics of the biodiesel were basically those recommended by the European Organization for Normalization (CEN), for its use in motor vehicles. Also the recommended standards for the EN 14214 norm were employed. Details of the procedures used can be found in previous papers $[10,19]$. Most experiments were done in triplicate, and standard deviations are shown numerically in tables or as error bars in figures.

\section{Results and Discussion}

Before starting the study of the influence of variables, prior experiments in order to determine the optimal position of the probe in the reaction medium were carried out. In these experiments the methanol:oil molar ratio was 9:1, the concentration of catalyst $(\mathrm{KOH})$ was $0.7 \%(w / w)$ and the power or amplitude of the probe was fixed at $40 \%$. Three different positions were investigated: the probe in the oil phase, the probe in the methanol-oil interphase and the probe in the methanol phase. It was observed that the degree of conversion is small when the probe was in the oil phase. The conversion increased when the probe was placed in the methanol-oil interphase, reaching the top value when the probe is in the methanol phase. These results can be due to the extent cavitational intensity generated as a consequence of the presence of the probe in the oil phase, methanol phase or in the methanol-oil interphase. These circumstances affected the physicochemical properties, mainly viscosity, surface tension and density. As it is established in literature, methanol favors the generation of cavitation conditions and, as a consequence, the maximum conversion was obtained in this case [15]. In view of the above, the probe was placed in the methanol phase in all the subsequent experiments.

The generation of microturbulence for the cavitation bubbles located in the proximity of the interphase methanol-oil originated an emulsion of the two liquids. The dispersion of methanol in the oil depended on the intensity of the microturbulences that were generated by the cavitation bubbles in methanol and vice versa. The intensity of the microturbulences depended on the physical properties of the liquid medium such as density, viscosity and surface tension and also the amplitude of the acoustic waves driving the bubble motion. The methanol and oil phases have different physical properties and, therefore, the intensity of the microturbulences in the two phases was different. Consequently, the extension of the dispersion of methanol in oil cannot be the same as the dispersion of oil in methanol. The uniform dispersion of methanol in the oil phase originated the necessary interfacial area for the reaction to take place. This dispersion was produced, as it has been indicated, by the high level of microturbulences generated by the cavitation of the bubbles of methanol in the proximity with 
the interphase and, as it was manifested by Kalva [18], the dispersion increased with the methanol:oil molar ratio. As a consequence, the influence of this variable on the process should be notable.

Another aspect to consider is the reaction temperature. In the experiments, heating was not applied, but it was verified that the reaction temperature increased, due to irradiation, achieving maximum temperatures of up to $80{ }^{\circ} \mathrm{C}$ (run 9). Naturally, the experimental results showed that, for low temperatures, the extension of the conversion was also low and when temperature increased, the conversion also increased, showing, to a certain extent, a positive impact of temperature. But it is necessary to consider other factors. An increase in temperature implied an increase of the solubility of methanol in the oil phase and, also, an increase of the reaction rate. These two factors contributed to increase the conversion of the reaction. But at high temperatures, the extension of the cavitational effects was dampened and, on the other hand, methanol leaked of the reaction medium to surpass the boiling temperature. Hence, there was an optimal temperature that is dependent on all the previous aspects [15].

\subsection{Effect of Ultrasound Power in Methyl Ester Conversion}

In order to reduce the production costs of biodiesel it is necessary to optimize the amount of energy supplied to the reaction mixture. The objective was to obtain a maximum yield and a high formation rate with the lowest energy consumption. In this sense, it is known that when the intensity of the radiation (i.e., ultrasound power/irradiation area) increased, a more violent collapse of the bubbles of cavitation took place, producing a bigger mixing intensity in the methanol-oil interphase. These circumstances originated the formation of a very fine emulsion that favored the mass-transfer coefficient, causing a high formation of biodiesel [7].

Power had an influence on the size and number of bubbles, maximum live time, pressure that break these bubbles, elevation of the temperature inside the liquid, generation of cavitational effects and over the final collapse intensity. Also the intensity blending depended on the density of energy. Thus, generally, high levels of power dissipation promoted cavitational effects, but at times, these depended on the geometry of the reactor. For this reason, an optimal value of power dissipation is often observed due to phenomena of acoustic disengaging [21]. As a result, for high powers, a damping effect, which originates a decrease in the energy transfer and a low cavitational activity, was observed [15].

The results obtained in the study of the ultrasonic power are shown in Figure 3. The power was varied from 20 to $100 \%$ (experiments 1 to 5). Also, in order to draw a comparison, an experiment (run 12), carried out at $55^{\circ} \mathrm{C}$ in isothermal conditions, with $0 \%$ of ultrasonic power, is enclosed. Also, in order to draw a comparison, an experiment (run 12), carried out at $55^{\circ} \mathrm{C}$ in isothermal conditions, with $0 \%$ ultrasonic power, is enclosed. In this experiment, a heating power of $500 \mathrm{~W}$ was supplied in order to get the indicated conditions. The conditions of this experiment were chosen considering that are standard and common conditions of transesterification processes carried out by conventional heating methods. There was a positive effect of power, so that, for a given reaction time, the conversion was greater as the power was higher. The curves showed an induction period that diminished when power increased. Differences in conversion and induction times were smaller as the power level increased so, for powers above $60 \%$, respective curves tended to an overlap situation. Thus, in the current situation, it took $15 \mathrm{~min}$ to achieve very high conversions (around 90\%), for the experiments carried out with an ultrasound power between 160 and $400 \mathrm{~W}$. An aspect that should be considered is the little variation of the maximum conversion with the applied power. In effect, runs 3, 4 and 5 lead to similar conversions even though the applied power differed considerably (60 to 100\%). In this sense, the observations of Shing et al. [12] should be considered, showing that at higher amplitudes, ester yields were drastically reduced. This was attributable to cracking followed by FAME oxidation to aldehydes, ketones, and lower-chained organic fractions. 


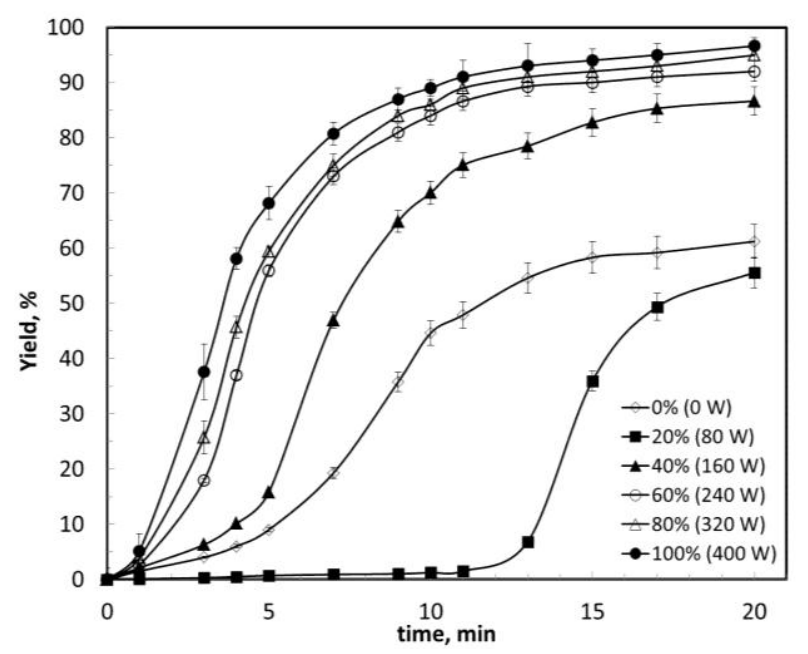

Figure 3. Effect of ultrasound power on the extent of conversion (MeOH:Oil 9:1, 0.7\% KOH).

In addition, Figure 4 shows the evolution of temperature with reaction time for ultrasound powers between 40 and 100\%. In all cases the same trend was observed: in the first minutes of reaction, the temperature increased very quickly, and later it stabilized at a constant value. The time required to achieve this constant value was between 3 and $4 \mathrm{~min}$, and the maximum values of temperature were proportional to the applied power $\left(60,64,68\right.$ and $70{ }^{\circ} \mathrm{C}$ for $40,60,80$ and $100 \%$ powers respectively). Therefore, a balance between the heat generated as a consequence of the ultrasound radiation and the heat lost through the walls of the reactor took place. The result is a process that occurs near the isothermal regime. Another aspect to consider is the energy consumption. Ultrasonic powers of $160-240 \mathrm{~W}$ gave $90 \%$ yields. These results are in accordance with other research data, with similar power ranges and over $90 \%$ yields [22]. However, thermal powers of $500 \mathrm{~W}$ only produced a $55 \%$ yield. This implies a considerable energy savings.

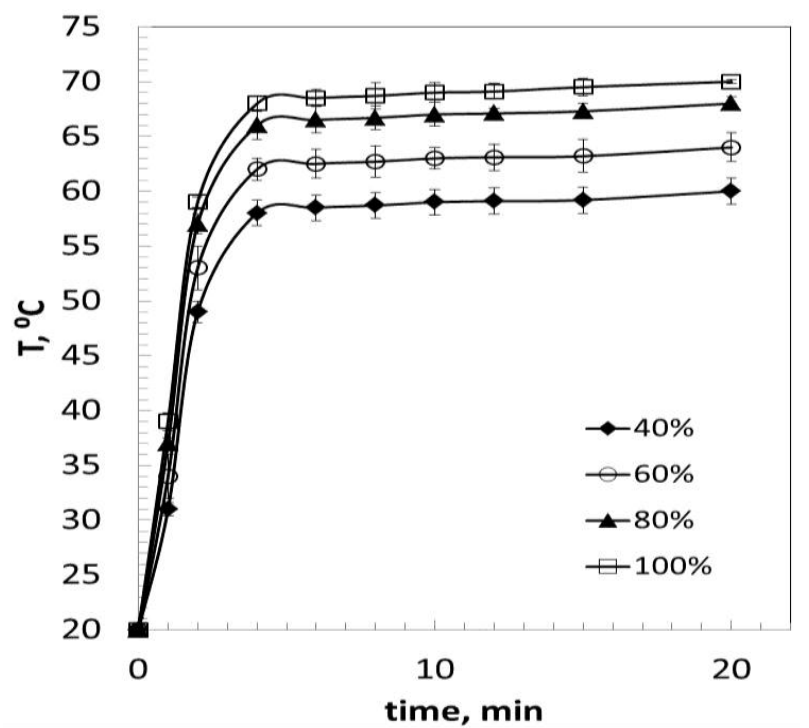

Figure 4. Temperature vs time. Effect of ultrasound power (methanol:oil 9:1, 0.7\% KOH).

\subsection{Effect of Catalyst Amount on Methyl Ester Conversion}

Figure 5 shows the influence of the catalyst amount (run 5 to 8 ). The positive effect of this variable on the reaction rate of the process can be easily seen. 


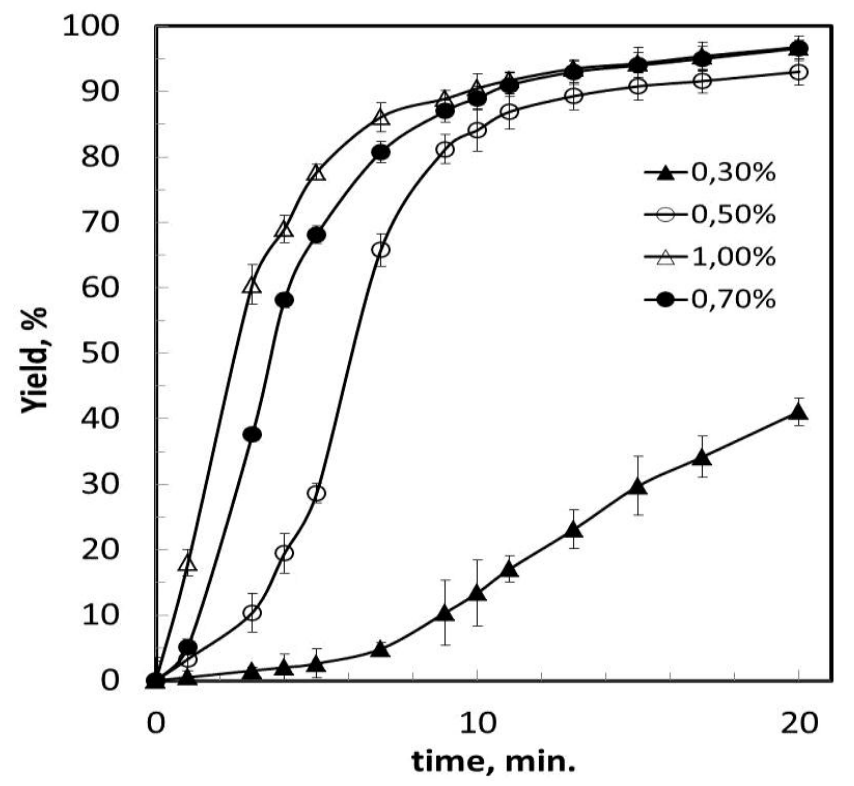

Figure 5. Effect of catalyst concentration on conversion (MeOH:Oil 9:1, 100\% ultrasound power).

For very low concentrations, there was a long induction period. At high concentrations this induction period did not exist. For concentrations of catalyst under $0.7 \%$, the conversion increased with concentration. Higher values of concentration lead to very similar conversions (run 5 and 8). This resulted in catalyst savings, which is positive from an economic point of view, and also facilitated the final product washing and/or the recovery of the catalyst, which is positive from a technical and environmental point of view.

Like in other works, it is possible to see two areas. In the first one, which can extend up to 7-12 $\mathrm{min}$, a positive influence on the amount of catalyst on the rate of formation of methyl esters was observed. Indeed, as the amount of catalyst increased, it reached peak production sooner, that is, the asymptotic zone of the curve. The second area of the curve (the asymptote) was very similar in all cases (except for $0.3 \%$ ).

Except for the experiment carried out with $0.3 \%$ catalyst (run 6) the short time necessary to achieve a maximum conversion was noticeable. In this sense, the ultrasound helps reduce the amount of catalyst needed due to the chemical activity due to cavitation. Thus, the necessary quantity of catalyst to achieve a given conversion was smaller when the reaction was carried out in the presence of ultrasound. A benefit was the resulting increase of the glycerol purity [23].

\subsection{Effect of Methanol:Oil Ratio on Methyl Ester Conversion}

One of the most important variables affecting the yield of esters is the alcohol:triglyceride molar ratio. As it is known, the stoichiometric ratio for transesterification requires three moles of alcohol and one mole of triglyceride to yield three moles of fatty acid alkyl esters and one mole of glycerol. However, transesterification is an equilibrium reaction in which a large excess of alcohol is required to drive the reaction to the right (products). However, the high molar ratio of alcohol to vegetable oil interferes with the separation of glycerin because there was an increase in solubility. When glycerin remained in solution, it helped to drive the equilibrium to the left (reactive), lowering the yield of esters [24]. Consequently, the methanol:oil molar ratio is a variable that should be optimized.

Figure 6 shows the results obtained (experiments 5, 9 to 11). Competing considerations are observed. On one hand, the higher initial reaction rate happened in the experiment with the lowest ratio of $3: 1$ and the lowest in the experiment with 12:1. On the other hand, the maximum conversion achieved was superior with increasing the methanol: oil molar ratio. 


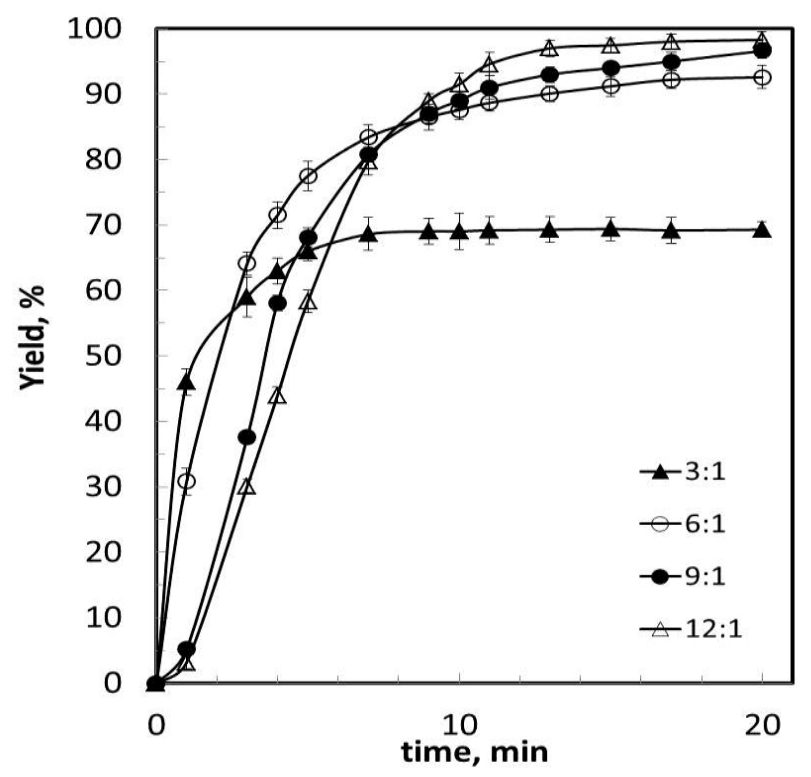

Figure 6. Effect of methanol:oil molar ratio on the extent of conversion $(0.7 \% \mathrm{KOH}, 100 \%$ ultrasound power).

This fact suggests that oil and methanol easily became an emulsion when there was a small amount of methanol, resulting in a higher rate of methanolysis. This implies that, under ultrasonic irradiation, small droplets of methanol were generated rapidly and easily achieved emulsion formation in the oil phase, leading to quick emulsion formation. In contrast, the droplet size of the emulsion was greater when there was a higher amount of methanol, and this resulted in a slower initial reaction rate. Furthermore, when there was a larger excess of methanol, the probability of the small size droplets of methanol encountering each other to agglomerate into larger droplets was larger than when there was a smaller excess of methanol. This trend towards large droplet formation may result in a slow transesterification reaction rate [25].

With an increase in the molar ratio, the quantity of methanol in the reaction mixture increased, which mainly affected the cavitation intensity. Excess of methanol provided additional cavitation events in the reactor, leading to the formation of enhanced emulsion quality (smaller drop sizes), providing additional area for the reaction and hence increased conversion [26].

Also, in connection with the previous figure, it can be noted that in the experiment with a molar ratio of 3:1, the reaction was stopped at $9 \mathrm{~min}$ due to the fast and excessive increase in temperature $\left(80^{\circ} \mathrm{C}\right.$ at the end), which made the control of this variable difficult. In addition to the above, the low effect of temperature in ultrasonic system is related to the collapse of bubbles caused by cavitations that produced intense local heating and high pressures, with very short lifetimes, which have a much higher effect than elevating the temperature of the liquid media. Furthermore, temperature affected the vapor pressure, surface tension, and viscosity of the liquid medium. While higher temperature increased the number of bubble cavitations, the collapse of bubbles was cushioned by the higher vapor pressure, reducing the effect of ultrasound application in the reaction [27].

\subsection{Biodiesel Properties}

Table 2 shows the yield, density and viscosity obtained for all the experiments. Table 3 shows other parameters of the biodiesel corresponding with the two experiments with the highest yield in methyl esters. For comparison, in Tables 2 and 3, the values of the EN-14214 standard have been added. As it can be seen in Table 2, only in some experiments, it was possible to achieve the minimum conversion to methyl esters, required by the standard EN 14214. However, most of the experiments reached values close to the requirements. Only in experiments in which the applied power, or catalyst concentration, or the molar ratio of methanol: oil were minimal, was the conversion low. 
Table 3. Properties of biodiesel produced from rapeseed oil in the best conditions of this study.

\begin{tabular}{cccc}
\hline Parameter & Run 5 & Run 10 & EN-14214 \\
\hline FAME content, \% & $96.6( \pm 0.8)$ & $97.4( \pm 1.2)$ & 96.5 \\
Water content, $\%$ & $0.06( \pm 0.01)$ & $0.06( \pm 0.01)$ & $<0.05$ \\
Saponification value, $\mathrm{g}_{\mathrm{KOH}} \cdot \mathrm{g}_{\text {oil }}{ }^{-1}$ & $190.6( \pm 0.8)$ & $188.9( \pm 0.6)$ & \\
Iodine value, $\mathrm{wt} \%$ & $106.9( \pm 0.3)$ & $106.1( \pm 0.1)$ & $\leq 120$ \\
Acidity Index, $\mathrm{mg}_{\mathrm{KOH}} \cdot \mathrm{g}_{\text {oil }}{ }^{-1}$ & $0.49( \pm 0.02)$ & $0.48( \pm 0.01)$ & $\leq 0.5$ \\
CFPP, ${ }^{\circ} \mathrm{C}$ & $-7( \pm 1)$ & $-7( \pm 1)$ & $\geq 120$ \\
Flash point, ${ }^{\circ} \mathrm{C}$ & $178( \pm 1)$ & $175( \pm 1)$ & \\
Combustion point, ${ }^{\circ} \mathrm{C}$ & $185( \pm 1)$ & $189( \pm 1)$ & \\
Cetane index & $44.1( \pm 0.2)$ & $43.8( \pm 0.3)$ & $860-900$ \\
Density $\left(\mathrm{kgm}^{-3}\right)$ & $861.9( \pm 1.1)$ & $870.9( \pm 0.8)$ & $3.5-5.0$ \\
Viscosity at $40{ }^{\circ} \mathrm{C}(\mathrm{cSt})$ & $4.7( \pm 0.2)$ & $4.9( \pm 0.2)$ &
\end{tabular}

In relation to density, the same ranges between 861.9 and $888.5 \mathrm{~kg} / \mathrm{m}^{3}$ were found, within the limits specified by the standard. Viscosity varied between 4.4 and 13.9 cSt. Except for the three experiments with low conversion, the presented viscosity values are in accordance with the standard. One overall effect was the relationship between conversion and viscosity: the more viscosity the less conversion. Consequently, most of the biodiesels obtained complied with the EN-14214 specifications, especially in those cases where the conversions obtained were high. For the other parameters (see Table 3), no significant differences were observed when high conversions were reached. Furthermore, it can be seen that, in general, the values of the standard were met.

\subsection{Kinetic Study}

As it has been indicated previously, the physical mechanism responsible for the beneficial action of ultrasound is the formation of a fine emulsion between oil and methanol that enhances the interface area for the reaction. No chemical effect of ultrasound, i.e., production of radical species and induction/acceleration of the reaction by these species, seemed to play any role [18]. In addition, the ultrasound radiation allowed one to get high temperature process in a short time. These temperatures (Figure 4) reached a maximum value ( 3 or $4 \mathrm{~min}$ ) and at a later time they were constant. Therefore, the reaction, in the presence of ultrasound radiation, was similar to an isothermal regime process.

Besides, the transesterification reaction was complex, because secondary reactions of saponification or neutralization can happen. Additionally, the initial heterogeneous character of the reaction made the process difficult $[28,29]$. In our case, rapeseed oil was refined, and the free acidity was small, that is why saponification or neutralization reactions did not happen. The concentration of the catalyst did not change to get the asymptotic zone of the curves, and we assumed that the reaction-formations of monoglycerides, diglycerides and triglycerides were very fast and that there was no intermediate of reaction. That is, the transesterification reaction was considered as an only and global reaction, therefore, considering the above and in line with the literature [30], the reaction would be represented by Equation (2), where TG is the triglyceride, $\mathrm{MeOH}$ is methanol, $\mathrm{ME}$ is methyl-esters and $\mathrm{G}$ is glycerin.

$$
\mathrm{TG}+3 \mathrm{MeOH} \leftrightarrow 3 \mathrm{ME}+\mathrm{G}
$$

The reaction (Equation (2)) is reversible, but in practice an excess of methanol was used and it can be considered irreversible. In addition, the kinetic study only was carried out until the curve showed the asymptotic trend. Under these conditions the inverse reaction lacks importance. The reaction rate is expressed by Equation (3), where $\alpha$ and $\beta$ are the orders of reaction in relation to triglycerides and methanol respectively, $\mathrm{k}^{\prime}$ is a constant that includes the effect of the catalyst, and $\mathrm{t}$ is the reaction time:

$$
-\mathrm{d}[\mathrm{TG}] / \mathrm{dt}=\mathrm{k}^{\prime}[\mathrm{TG}]^{\alpha}[\mathrm{MeOH}]^{\beta}
$$


Equation (3) can be rewritten like Equation (4), where $\mathrm{k}$ regroups $\mathrm{k}^{\prime}$ and the concentration of methanol. This concentration can be considered constant because a very large concentration of methanol was used:

$$
-\mathrm{d}[\mathrm{TG}] / \mathrm{dt}=\mathrm{k}[\mathrm{TG}]^{\alpha}
$$

Expressing the concentration of triglycerides in terms of conversion $(\mathrm{X})$, Equation (4) takes the appearance of Equation (5), where $\mathrm{TG}_{0}$ represents the initial concentration of triglycerides:

$$
\mathrm{dX} / \mathrm{dt}=\mathrm{k}\left[\mathrm{TG}_{0}\right](1-\mathrm{X})^{\alpha}
$$

The integration of (5), with the most widely-used hypothesis in literature (pseudo first order kinetic model), that is, with $\alpha=1$, leads to Equation (6):

$$
\ln (1-X)=-\mathrm{kt}
$$

Equation (1) has been applied to Experiments 2, 3, 4 and 5, in which the ultrasound power varied. In these experiments, the methanol:oil molar ratio was always 9:1 and the catalyst concentration $0.7 \%$. In Table 4 , the pseudo kinetic constants and $\mathrm{R}^{2}$ coefficient, obtained by means of regression analysis of Equation (1) are shown.

Table 4. Kinetic analysis of the transesterification process.

\begin{tabular}{cccc}
\hline Temperature, ${ }^{\circ} \mathbf{C}$ & Ultrasound Power (\%) & Pseudo First-Order Kinetic, $\mathbf{m i n}^{-\mathbf{1}}$ & $\mathbf{R}^{\mathbf{2}}$ \\
\hline 60 & 40 & 0.1669 & 0.98 \\
64 & 60 & 0.1872 & 0.98 \\
68 & 80 & 0.2103 & 0.98 \\
70 & 100 & 0.2170 & 0.99 \\
\hline
\end{tabular}

The temperature shown for each experiment was the temperature that the system reached after 3 or 4 min (final temperature). The high values of $\mathrm{R}^{2}$ confirmed that this system followed a pseudofirst kinetic model. The relationship among the specific reaction rate constant $(\mathrm{k})$, absolute temperature (T) and activation energy (Ea) is given by Arrhenius Equation (7), where A is the frequency factor and $R$ is the universal gas constant:

$$
\mathrm{k}=\mathrm{Aexp}(-\mathrm{Ea} / \mathrm{RT})
$$

The linear regression of Equation (7) provides the value of the activation energy. The best fit is shown in Equation (8). These data lead to a value of $\mathrm{Ea}=25.51 \mathrm{~kJ} / \mathrm{mol}$. This value was relatively small, which reveals a great catalytic activity of the basic catalysts $(\mathrm{KOH})$ :

$$
\operatorname{lnk}=-3068.5 / \mathrm{T}+7.428 ; \mathrm{R}^{2}=0.993
$$

The activation energy was similar to that obtained in rapeseed oil transesterification in the presence of co-solvents $(21.88 \mathrm{~kJ} / \mathrm{mol})$ [31] and slightly lower than in the case of Jatropha curcas transesterification by ultrasound (31.29 and $57.33 \mathrm{~kJ} / \mathrm{mol}$ ) [32]. On the other hand, the activation energy in other catalysis-base transesterification through thermal heat were superior. For instance, for palm oil transesterification an activation energy of $105 \mathrm{~kJ} / \mathrm{mol}$ was found [33].

\section{Conclusions}

The suitability of the use of ultrasound for rapeseed oil transesterification was studied. The main findings of this research were:

Ultrasonic irradiation facilitated the rapeseed transesterification, since high biodiesel yields were achieved after short reaction times (20 $\mathrm{min})$. It was observed, during experimentation, that the temperature of the reaction mixture increased, as a result of ultrasound radiation. 
The use of ultrasound radiation did not need additional heating, what can suppose an energy savings. Nevertheless, it is necessary to consider the energy consumed in the generation of ultrasound.

The influence of the ultrasound power and the catalyst concentration was positive, so the yield of the process and the reaction rate increased as these variables increased. The methanol:oil molar ratio also lead to a bigger yield of the process, but the lower values of the methanol:oil molar ratio lead to the biggest reaction rate.

The obtained results showed that ultrasonic powers of $320 \mathrm{~W}$, a catalyst concentration of $0.7 \%$ and a methanol:oil molar ratio of 9:1 are enough to achieve biodiesel yields of $95 \%$. These conditions can be considered adequate to carry out the process. The characteristics of the biodiesel, determined by the EN 14214 standard, revealed that, in general, they met the established limits. The final product had similar properties to a diesel-oil.

The transesterification reactions followed a pseudo-first order kinetic model and the rate constants at several temperatures were determined. Also, the activation energy was determined by the Arrhenius equation.

Author Contributions: Conceptualization, J.M.E., A.P. and N.S.; Methodology, J.M.E., A.P., N.S. and S.N.; Validation, J.M.E., A.P., N.S. and S.N.; Formal Analysis, A.P. and N.S.; Investigation, A.P. and N.S.; Resources, J.M.E., A.P., N.S. and S.N.; Data Curation, J.M.E., A.P., N.S. and S.N.; Writing-Original Draft Preparation, J.M.E., A.P., N.S. and S.N.; Writing-Review \& Editing, J.M.E. and S.N.; Supervision, J.M.E. and S.N.

Acknowledgments: The authors would like to thank the "Junta de Extremadura" ("Ayudas para la realización de actividades de investigación y desarrollo tecnológico, de divulgación y de transferencia de conocimiento por los Grupos de Investigación de Extremadura") and the FEDER "Fondos Europeos de Desarrollo Regional" for the financial support received.

Conflicts of Interest: The authors declare no conflict of interest.

\section{References}

1. Caldeira-Pires, A.; da Luz, S.M.; Palma-Rojas, S.; Oliveira Rodrigues, T.; Chaves Silverio, V.; Vilela, F.; Barbosa, P.C.; Alves, A.M. Sustainability of the biorefinery industry for fuel production. Energies 2013, 6, 329-350. [CrossRef]

2. Guo, M.; Song, M.; Buhain, J. Bioenergy and biofuels: History, status, and perspective. Renew. Sustain. Energy Rev. 2015, 42, 712-725. [CrossRef]

3. Ma, F.; Hanna, M.A. Biodiesel production: A review. Bioresour. Technol. 1999, 70, 1-15. [CrossRef]

4. Pinzi, S.; Garcia, I.L.; Lopez-Gimenez, F.J.; Luque de Castro, M.D.; Dorado, G.; Dorado, M.P. The Ideal Vegetable Oil-based Biodiesel Composition: A Review of Social, Economical and Technical Implications. Energy Fuel 2009, 23, 2325-2341. [CrossRef]

5. Jurac, Z.; Zlatar, V. Optimization of raw material mixtures in the production of biodiesel from vegetable and used frying oils regarding quality requirements in terms of cold flow properties. Fuel Proc. Technol. 2013, 106, 108-113. [CrossRef]

6. Azad, A.K. Biodiesel from Mandarin Seed Oil: A surprising source of alternative fuel. Energies 2017, 10, 1689. [CrossRef]

7. Mahamuni, N.N.; Adewuyi, Y.G. Optimization of the Synthesis of Biodiesel via Ultrasound-Enhanced Base-Catalyzed Transesterification of Soybean Oil Using a Multifrequency Ultrasonic Reactor. Energy Fuels 2009, 23, 2757-2766. [CrossRef]

8. Demirbas, A. Progress and recent trends in biodiesel fuels. Energy Convers. Manag. 2009, 50, 14-34. [CrossRef]

9. Ramachandran, K.; Suganya, T.; Nagendra Gandhi, N.; Renganathan, S. Recent developments for biodiesel production by ultrasonic assist transesterification using different heterogeneous catalyst: A review. Renew. Sustain. Energy Rev. 2013, 22, 410-418. [CrossRef]

10. Encinar, J.M.; González, J.F.; Pardal, A. Transesterification of castor oil under ultrasonic irradiation conditions. Preliminary results. Fuel Proc. Technol. 2012, 103, 9-15. [CrossRef]

11. Singh, A.K.; Fernando, S.D.; Hernandez, R. Base-Catalyzed Fast Transesterification of Soybean Oil Using Ultrasonication. Energy Fuels 2007, 21, 1161-1164. [CrossRef]

12. Hanh, H.D.; Dong, N.T.; Okitsu, K.; Nishimura, R.; Maeda, Y. Biodiesel production through transesterification of triolein with various alcohols in an ultrasonic field. Renew. Energy 2009, 34, 766-768. [CrossRef] 
13. Ji, J.; Wang, J.; Li, Y.; Yu, Y.; Xu, Z. Preparation of biodiesel with the help of ultrasonic and hydrodynamic cavitation. Ultrasonics 2006, 44, e411-e414. [CrossRef] [PubMed]

14. Veljković, V.B.; Avramović, J.M.; Stamenković, O.S. Biodiesel production by ultrasound-assisted transesterification: State of the art and the perspectives. Renew. Sustain. Energy Rev. 2012, 16, 1193-1209. [CrossRef]

15. Hingu, S.M.; Gogate, P.R.; Rathod, V.K. Synthesis of biodiesel from waste cooking oil using sonochemical reactors. Ultrason. Sonochem. 2010, 17, 827-832. [CrossRef] [PubMed]

16. Talebian-Kiakalaieh, A.; Amin, N.A.S.; Mazaheri, H. A review on novel processes of biodiesel production from waste cooking oil. Appl. Energy 2013, 104, 683-710. [CrossRef]

17. Santos, F.F.P.; Rodrigues, S.; Fernandes, F.A.N. Optimization of the production of biodiesel from soybean oil by ultrasound assisted methanolysis. Fuel Proc. Technol. 2009, 90, 312-316. [CrossRef]

18. Kalva, A.; Sivasankar, T.; Moholkar, V.S. Physical mechanism of ultrasound-assisted synthesis of biodiesel. Ind. Eng. Chem. Res. 2009, 48, 534-544. [CrossRef]

19. Encinar, J.M.; Pardal, A.; Martínez, G. Transesterification of rapeseed oil in subcritical methanol conditions. Fuel Process. Technol. 2012, 94, 40-46. [CrossRef]

20. Martínez, G.; Sánchez, N.; Encinar, J.M.; González, J.F. Fuel properties of biodiesel from vegetable oils and mixtures. Influence of methyl esters distribution. Biomass Bioenergy 2014, 63, 22-32. [CrossRef]

21. Gole, V.L.; Gogate, P.R. A review on intensification of synthesis of biodiesel from sustainable feed stock using sonochemical reactors. Chem. Eng. Process. 2012, 53, 1-9. [CrossRef]

22. Sheng Ho, W.W.; Kiat Ng, H.; Gan, S. Advances in ultrasound-assisted transesterification for biodiesel production. Appl. Therm. Eng. 2016, 100, 553-563.

23. Kumar, D.; Kumar, G.; Poonam; Singh, C.P. Fast, easy ethanolysis of coconut oil for biodiesel production assisted by ultrasonication. Ultrason. Sonochem. 2010, 17, 555-559. [CrossRef] [PubMed]

24. Meher, L.C.; Vidya Sagar, D.; Naik, S.N. Technical aspects of biodiesel production by transesterification-A review. Renew. Sustain. Energy Rev. 2006, 10, 248-268. [CrossRef]

25. Thanh, L.T.; Okitsu, K.; Sadanaga, Y.; Takenaka, N.; Maeda, Y.; Bandow, H. Ultrasound-assisted production of biodiesel fuel from vegetable oils in a small scale circulation process. Bioresour. Technol. 2010, 101, 639-645. [CrossRef] [PubMed]

26. Deshmane, V.G.; Gogate, P.R.; Pandit, A.B. Ultrasound-Assisted Synthesis of Biodiesel from Palm Fatty Acid Distillate. Ind. Eng. Chem. Res. 2009, 48, 7923-7927. [CrossRef]

27. Santos, F.F.P.; Malveira, J.Q.; Cruz, M.G.A.; Fernandes, F.A.N. Production of biodiesel by ultrasound assisted esterification of Oreochromis niloticus oil. Fuel 2010, 89, 275-279. [CrossRef]

28. Veljkovic, V.B.; Stamenkovic, O.S.; Todorovic, Z.B.; Lazic, M.L.; Skala, D.U. Kinetics of sunflower oil methanolysis catalyzed by calcium oxide. Fuel 2009, 88, 1554-1562. [CrossRef]

29. Stamenkovic, O.S.; Todorovic, Z.B.; Lazic, M.L.; Veljkovic, V.B.; Skala, D.U. Kinetics of sunflower oil methanolysis at low temperatures. Bioresour. Technol. 2008, 99, 1131-1140. [CrossRef] [PubMed]

30. Ramezami, K.; Rowshanzamir, S.; Eikani, M.H. Castor oil transesterification reaction: A kinetic study and optimization of parameters. Energy 2010, 35, 4142-4148. [CrossRef]

31. Encinar, J.M.; Pardal, A.; Sánchez, N. An improvement to the transesterification process by the use of co-solvents toproduce biodiesel. Fuel 2016, 166, 51-58. [CrossRef]

32. Choudhury, H.A.; Srivastava, P.; Moholkar, V.S. Single-step ultrasonic synthesis of biodiesel from crude Jatropha curcas oil. AIChE J. 2014, 60, 1572-1581. [CrossRef]

33. Permsuwan, A.; Tippayawong, N.; Tanongkiat, T.; Thararux, C.; Wangkarn, S. Reaction Kinetics of Transesterification between Palm Oil and Methanol under Subcritical Conditions. Energy Sci. Technol. 2011, 2, 35-42.

(C) 2018 by the authors. Licensee MDPI, Basel, Switzerland. This article is an open access article distributed under the terms and conditions of the Creative Commons Attribution (CC BY) license (http:/ / creativecommons.org/licenses/by/4.0/). 